\title{
La construcción discursiva del joven trabajador: Un análisis crítico a los informes Tendencias Mundiales de Empleo*
}

\section{The Discursive Construction of the Youth as Workers: A Critical Analysis of Global Employment Trends for Youth}

Recepción: 03 Agosto 2015 | Aprobación: 15 Junio 2017

\author{
Guillermo Rivera-Aguilera ${ }^{a}$ \\ Pontificia Universidad Católica de Valparaíso, Chile \\ ORCID: http://orcid.org/0000-0002-2724-481X
}

a Autor de correspondencia. Correo electrónico: griverov@gmail.com

Para citar este artículo: Rivera-Aguilera, G. (2018). La construcción discursiva del joven trabajador: Un análisis crítico a los informes Tendencias Mundiales de Empleo. Universitas Psychologica, 17(1), 1-14 https://doi.org/10.11144/Javeriana.upsy17-1.cdjt

\section{RESUMEN}

El presente artículo se propone como objetivo describir las formas en que la Organización Internacional del Trabajo (OIT) construye discursivamente al joven trabajador. Para esto, se realiza un análisis de discurso de los informes Tendencias Mundiales de Empleo (TEM), publicados por la OIT entre 2004-2014. Los resultados obtenidos dan cuenta que en los informes TEM aparecen seis figuras de la subjetividad que construyen al joven trabajador: a) el joven pobre, b) el joven ocioso, c) la generación perdida, d) el joven feliz, e) los desafortunados jóvenes, y f) los jóvenes desconfiados. De acuerdo al análisis basado en el enfoque de la Gubernamentalidad, se puede constatar que estas formas de conceptualizar a la juventud se fundamentan desde una lógica economicista, basada en valores neoliberales. Se discute en torno a cómo estas figuras de la subjetividad corresponden a Tecnologías del Yo, que permiten prescribir formas de conducir la conducta de los jóvenes para su inclusión al mercado del trabajo.

Palabras clave

gubernamentalidad; OIT; juventud; empleo; análisis del discurso.

\footnotetext{
ABSTRACT

This article aims to describe the ways in which the International Labour Organization (ILO) discursively constructs the youth as workers. To do this, a discourse analysis of the Global Empoyment Trends for Youth (GET) reports, published by the ILO from 2004 to 2014, was conducted. The results show six figures of subjectivity that construct the young workers in the TEM reports: a) the poor youth, b) the idle youth, c) the lost generation, d) the happy youth, e) the unfortunate youth, f) the distrust youth. According to the Governmentality approach, it can be seen how these ways of conceptualizing young workers correspond to neoliberal values. Its discussed how these figures of subjectivity correspond to Technologies of the Self, that allow conduct the conduct of youth in order to prescribe way of including young people into the labor market.

Keywords

governmentality; ILO; youth; employment; discourse analysis.
} 
La crisis económica del 2008 ha tenido como consecuencia un aumento en el desempleo y en la precariedad laboral de los jóvenes a escala global (OIT, 2010, 2013). De acuerdo a datos oficiales de la Organización Internacional del Trabajo (OIT), durante el año 2012 era tres veces más probable que perdiera el trabajo un joven antes que un adulto (OIT, 2013). Estos hechos, sumados al panorama de las transformaciones del trabajo y el empleo de las últimas décadas, registran niveles nunca antes vistos de desempleo juvenil a nivel global ${ }^{1}$ (OIT, 2013, 2012; PulidoMartínez \& Sato, 2013; Sennett, 2013).

Desde organizaciones internacionales, se advierte como legado de esta crisis 'una generación en peligro' de jóvenes que abandona su posibilidad de transformarse en 'sujeto productivo' para el mercado (Assusa \& Brandán, 2014; OIT, 2010, 2012). En este escenario, las políticas globales, encabezadas por organizaciones como el $\mathrm{G}_{2} \mathrm{O}^{2}$, la $\mathrm{OIT}^{3} \mathrm{y}$ el Banco Mundial ${ }^{4}$, han definido que es un tema prioritario "evitar el riesgo de que una proporción cada vez mayor de la población pierda la confianza en la economía global" (OIT, 2013, p. 6).

En los últimos años, estos altos índices de desempleo y desocupación han generado una serie de articulaciones provenientes de movimientos sociales asociados a la juventud, que a partir del 2011 se han desarrollado principalmente en España $(15 \mathrm{M})^{5}$, Grecia e Italia. Los jóvenes de los países mencionados han utilizado la protesta social pacifica como forma de demandar cambios estructurales al sistema económico, basado una retórica anticapitalista y antiglobalización (Chertkovskaya, Watt, Tramer, \& Spolestra, 2013).

Este artículo se detiene en profundizar cómo los efectos de la crisis económica han generado una variación discursiva en la Organización Internacional del Trabajo, respecto a la problemática del empleo-desempleo juvenil, así como en las formas de conceptualizar al joven trabajador. El hecho de profundizar en estas conceptualizaciones, diagnósticos y metáforas, respecto al grupo etario en cuestión, puede contribuir al entendimiento de una serie de políticas y dispositivos globales cuyo fin es promocionar el empleo juvenil (Miller \& Rose, 2009). Esto, en un momento donde las transformaciones del trabajo están marcando el presente y el futuro de las políticas de empleo a nivel global y a nivel local (Sisto, 2012).

De acuerdo a la literatura revisada, las estrategias desarrolladas por las políticas globales para hacer frente al problema, apuntan al diseño y a la generación de programas homogéneos, con características similares para contrarrestar la problemática del desempleo juvenil (Chertkovskaya et al., 2013; Dean, 1995; Fejes, 2010; Garsten \& Jacobsson, 2013; Holmqvist, Maravelias, \& Skalen, 2012). De hecho, se puede constatar cómo las implementaciones locales de programas de empleo son similares en países tan distintos como Suecia, Argentina, Holanda y Australia (Hopman, Winter, \& Koops, 2014; Assusa \& Brandán, 2014; Holmqvist et al., 2012; Ainswoth, 2001). Estas políticas tienen como eje transversal, una retórica basada en la construcción de un 'sujeto empleable', desde el cual el joven a través, de su participación en programas gubernamentales, es formado y/o capacitado para autogestionarse y responsabilizarse a sí mismo de su inclusión al mundo del trabajo (Assusa \& Brandán, 2014; Chertkovskaya et al., 2013; Holmqvist et al., 2012).

Si bien la literatura se ha concentrado en definir una serie de categorías de la juventud como 'sujeto empleable' (Garsten \& Jacobsson, 2013), este estudio se interesa por explorar otras formas de comprender la construcción del joven trabajador desde una perspectiva global. Es a partir de la revisión bibliográfica basada en los estudios antes mencionados, así como en la revisión de conceptualizaciones existentes sobre la juventud en organismos internacionales, que este artículo se plantea la siguiente pregunta: ¿Cómo se construye el joven trabajador en los documentos de la Organización Internacional del Trabajo? 
Para responder a esto, se toman como referencia los siete informes "Tendencias Mundiales de Empleo Juvenil" (TEM), que hasta la fecha han sido publicados por la OIT (OIT, 2004, 2006, 2008, 2010, 2012, 2013, 2014). Estos textos se caracterizan por ser declarativos respecto a la política de juventud y empleo a nivel global. Se elaboran desde el año 2004, con base en datos estadísticos que desarrolla la OIT, los cuales permiten diagnosticar las condiciones del mercado del trabajo para los jóvenes (OIT, 2004). Estos informes abarcan la década 2004-2014, hecho que permite analizar las repercusiones de la crisis económica en las políticas globales sobre juventud y empleo.

Este artículo se centra en la Organización Internacional del Trabajo, porque corresponde a la entidad reguladora del trabajo a nivel global. Desde su misión institucional, se plantea como objetivos: a) fomentar los derechos laborales, b) ampliar las oportunidades de acceder a un empleo decente, mejorar la protección social, c) fortalecer el diálogo al abordar temas relacionados con el trabajo (OIT, 2017) ${ }^{6}$. Por otra parte, corresponde a una institución de carácter global, que prescribe lo que algunos autores han definido como 'políticas sociales globales' (Kaasch, 2013). Estas formas de construcción de políticas definen una serie de conceptualizaciones, metas, normas, métodos, e instrumentos para ser aplicados a niveles locales (Kaasch, 2013), promoviendo, de esta maner, una serie de prácticas de autoformación, orientaciones y conductas morales de los individuos, definiendo sus derechos y obligaciones (Dean, 1995).

Conceptualmente, este estudio se sitúa desde el enfoque de la Gubernamentalidad. Como bien se ha descrito en una serie de publicaciones, los 'Estudios de la Gubernamentalidad' tienen sus orígenes en las ideas sobre el poder y las formas de gobierno de las poblaciones desarrolladas por Michel Foucault en los cursos 'Seguridad Territorio y Población', en 1978 (Foucault, 2009) y 'El Nacimiento de la Biopolítica' en 1979 (Foucault, 2012), impartidos en el Collège de France (Burchell, Gordon, \& Miller, 1991; Castro-Gómez, 2010; Flew, 2014; Grinberg,
2007; Miller \& Rose, 2009; Rose, O’Malley, \& Valverde, 2012; Senellart, 2009; VargasMonroy \& Pujal, 2013). Si bien los estudios de Gubernamentalidad fueron inicialmente cuestionados por su poca aplicación empírica, y por tener características eurocéntricas, en los últimos años se han desarrollado una serie de investigaciones sobre el trabajo y el empleo desde este enfoque (Rose, 1996; Grinberg, 2007; Vargas-Monroy \& Pujal, 2013; Mussetta, 2009). Estos estudios empíricos se desarrollan principalmente desde el mundo anglosajón (Chertkovskaya et al., 2013) y escandinavo (Diedrich \& Styhre, 2013; Fejes, 2010; Garsten $\&$ Jacobsson, 2013; Vesterberg, 2013; Holmqvist et al., 2012), y existe una corriente emergente desde el Latinoamérica (Assusa \& Brandán, 2014; Brandán, 2014; Castro-Gómez, 2010; Pulido-Martínez, 2012, 2008; Sáenz \& Granada, 2013; Vargas-Monroy \& Pujal, 2013).

El hecho de analizar cómo se construye una Gubernamentalidad global respecto a la juventud y el empleo, a partir del análisis de los discursos que elabora la OIT es la propuesta que explora este artículo. Para esto, se profundiza en los discursos que definen formas de control y normalización de la juventud, desarrollados por la OIT. Siguiendo las ideas de Foucault (2009) sobre la Gubernamentalidad, las políticas de empleo juvenil se desarrollan desde una racionalidad centrada en conducir la conducta de los jóvenes, esto con la finalidad de hacer que ejerzan su libertad de modos específicos, basados en los objetivos estratégicos de programas de empleo y del mercado del trabajo (Castro Gómez, 2010; Du Gay, 1996; Stecher \& Godoy, 2014).

En relación a lo expuesto, este estudio entiende las políticas públicas orientadas al empleo juvenil como el resultado de un accionar de las instituciones que interpelan al joven como trabajador, y que, en determinado momento, prescriben formas de diagnosticar e intervenir a la juventud. Tal como ha señalado Fejes (2010), estos criterios pueden ser analizados desde documentos oficiales de uso público. Para esto, se desarrolla un análisis documental (Atkinson \& Coffey, 2006; Prior, 2006), tomando como referencia los informes TEM de la de la 
OIT. Lo que se presenta a continuación es el resultado del análisis documental mencionado.

\section{Método}

Para ilustrar cómo se construyen los discursos sobre el joven trabajador desde la OIT, se realizó un Análisis Crítico del Discurso de los informes TEM (Parker, 2012, 2013; Fairclough, 2010; Fairclough \& Wodak, 2000; Stecher, 2010, 2014). Para esto, se utilizó como técnica el Análisis Documental (Atkinson \& Coffey, 2006; Prior, 2006, 2008), desde la cual se entenderán los informes TEM como actores que legitiman autoridad y validan formas de poder, permitiendo estandarizar y categorizar a la juventud en relación al empleo (Rivera-Aguilera, 2017).

Para este estudio, se seleccionó como corpus todos los informes TEM (OIT, 2004, 2006, 2008, $2010,2012,2013,2014)$, ya que resultan fuente de gran riqueza para mostrar el funcionamiento de la retórica justificativa con la cual se instalan transformaciones en política pública, interpelando las identidades de los actores sociales involucrados (Sisto \& Fardella, 2011).

En términos de procedimiento, la revisión documental del corpus implicó que surgieran una serie de categorías emergentes, las cuales permitieron dar cuenta de seis tipos de figuras normativas en torno a la construcción de la subjetividad juvenil. Los resultados se presentan desde dos ejes. El primero 'descriptivosecuencial' da cuenta cómo la OIT va cambiando la conceptualización del joven trabajador en la última década (2004-2014). En este eje se muestra cómo se clasifica la población juvenil desde seis maneras: a) La juventud pobre, b) la juventud ociosa, c) la generación perdida, d) la juventud feliz, e) los desafortunados jóvenes, y f) los desconfiados jóvenes. Para ejemplificar este proceso secuencial de construcción discursiva de la juventud, se presentan seis citas de los informes TEM, ordenadas cronológicamente. El segundo eje 'comparativo' describe cómo hay un cambio en los discursos de la OIT, basado en un antes y después de la crisis económica. El hecho de entender la crisis económica como punto de inflexión permite conocer diferencias discursivas que están presentes en los informes TEM. Finalmente, se desarrolla una discusión de cómo estos resultados contribuyen a responder la pregunta de investigación.

\section{Resultados}

\section{La clasificación de la población juvenil}

Tal como se ha mencionado, se revisarán seis formas de categorización de la juventud que emergen desde la revisión secuencial de los informes TEM (2004-2014).

\section{La juventud pobre}

Esta primera categoría que se presenta, se desarrolla en el primer informe TEM (2004), elaborado por la OIT. En términos genéricos, este informe plantea una conceptualización positiva y esperanzadora de la juventud desde la perspectiva de que este grupo etario constituye el futuro de nuestras sociedades. También sostiene que el empleo es el gran vehículo y la única alternativa para que los jóvenes puedan salir de la pobreza. A continuación, se presenta una cita que da cuenta de cómo se está entendiendo la problemática del desempleo juvenil en el año 2004, así como el rol y el compromiso de la OIT en esta tarea:

El vínculo entre desempleo juvenil y exclusión social está claramente comprobado. La incapacidad de encontrar empleo genera una sensación de exclusión e inutilidad entre los jóvenes y puede aumentar su participación en actividades ilegales. Hoy día, para muchos jóvenes estar sin trabajo significa no tener la oportunidad de salir de la pobreza. Además, se ha comprobado que la experiencia anterior de desempleo influye en las oportunidades futuras de empleo. En cualquier caso, el desempleo declarado es sólo parte del problema; aun cuando los jóvenes tengan empleo, las condiciones de trabajo pueden ser inadecuadas. Tanto en las economías industrializadas como en los países en desarrollo, los jóvenes tienen más probabilidades de encontrar trabajo 
intermitente (temporal, a tiempo parcial, eventual) e inseguro, muchas veces en la economía informal y con limitada protección laboral.

Desde su creación en 1919, la OIT ha tratado de mejorar las condiciones de empleo y trabajo juvenil. Hoy, impulsada por un nuevo sentimiento de urgencia a nivel mundial para buscar soluciones a las dificultades de empleo de los jóvenes, la OIT está reforzando su compromiso para facilitar, coordinar y ofrecer orientación técnica en un programa de trabajo integrado sobre el empleo de los jóvenes. Un elemento fundamental de este trabajo es el papel de liderazgo de los interlocutores sociales para promover el trabajo decente y la erradicación de la pobreza. (...) El empleo de los jóvenes forma parte integrante de la Declaración del Milenio y, al mismo tiempo, representa una contribución decisiva para alcanzar los otros Objetivos del Milenio, en particular los relacionados con la reducción de la pobreza. (OIT, 2004. p. 4)

De acuerdo con la cita, podemos constatar una clara relación entre desempleo juvenil y la exclusión social y la pobreza. A partir de esto, se puede entender que el joven pobre es aquel que no tiene trabajo, es un joven que se siente excluido e inútil por no trabajar. Si bien se plantea un claro cuestionamiento a las condiciones y formas de trabajo de los jóvenes que tienen empleo, es claro que el desempleo se asocia a la pobreza, y por lo tanto a jóvenes que tienen orígenes en países pobres o en vías de desarrollo. También podemos ver que los jóvenes de países industrializados tendrían mejores posibilidades de encontrar trabajo, es decir a mayor acceso que los jóvenes de países pobres. En relación a esto, se puede dar cuenta que en el año 2004, hay una clara diferencia para entender la problemática del desempleo juvenil entre economías avanzadas y países pobres.

Por otro lado, se puede ver el rol histórico de la OIT como institución global en el tema del empleo y el trabajo juvenil, ya que desde 1919 se plantea como un organismo internacional comprometido en la mejora de las condiciones del empleo juvenil en el mundo. A partir de esto, se puede sostener que la OIT se posiciona como la institución que tiene los conocimientos y la experiencia para contribuir con la resolución de la problemática del empleo-desempleo a nivel global. En la primera cita, es posible dar cuenta cómo se construye una 'juventud pobre', a la cual es urgente darle trabajo para que salga de su situación de pobreza. El mandato de la OIT es que no deben existir jóvenes pobres, es decir, la pobreza es un fenómeno que se debe erradicar. Por lo tanto, podemos entender la juventud pobre como una categoría que corresponde a un 'otro', que debe ser guiado y conducido desde una lógica de pastoreo (Foucault, 2009).

Desde esta perspectiva, el joven pobre será conducido con base en lineamientos de organismos internacionales, planteados principalmente desde los Objetivos del Milenio. Estas políticas sociales globales permitirán al joven pobre dejar su condición de 'otro', desde una intervención programática que le proporcionará 'trabajo decente' como forma de sacarlo de la pobreza, y desde ahí, su inclusión social y económica. Desde lo expuesto, se puede entender que hay una institucionalidad global que sabe cómo resolver la problemática (saber experto) que articula y coordina acciones para que el joven se incorpore al mercado del trabajo y deje de ser pobre.

\section{La juventud ociosa es costosa}

En el informe TEM del año 2006, se va reforzando una perspectiva económica en la forma de plantear la problemática del empleodesempleo. Se llega a plantear, desde una perspectiva global, que el joven que no trabaja es un ciudadano costoso, ya que no contribuye al bienestar de los países. A continuación, presentamos un fragmento que da cuenta de esto:

La segunda ganancia obvia de recapturar el potencial productivo de la juventud subutilizada es una ganancia económica. La juventud ociosa es costosa. No contribuyen al bienestar económico del país - al contrario. La pérdida de ingreso en la generación más joven se traduce en una falta de ahorros así como una pérdida de demanda agregada. Algunos jóvenes que no pueden ganarse la vida tienen que ser 
mantenidos por sus familias lo que disminuye la cantidad de dinero que queda para gastar e invertir a nivel de hogar. Las sociedades pierden la inversión que llevaron a cabo en la educación y los gobiernos no reciben contribuciones a sus sistemas de seguridad social y se ven obligados a gastar más en servicios remédiales, incluyendo programas de prevención del uso de drogas y del crimen. Todo esto amenaza el potencial de desarrollo de las economías. Por lo tanto, tiene sentido para un país enfocarse en la juventud desde un punto de vista de costo-beneficio. (OIT, 2006. p. 6)

En esta segunda cita, se plantea la juventud trabajadora como una ganancia económica para los países. A partir de esta afirmación, se entiende costosa una juventud inactiva, constituye una pérdida de la inversión del Estado en términos educativos y de seguridad social. Llama la atención que en el fragmento seleccionado, el joven que no trabaja se piscologiza o patologiza en términos de salud mental, asociándolo al consumo de drogas y al crimen. El discurso de la OIT apela a hacer frente a la problemática del empleo-desempleo desde un argumento economicista, ya que corresponde a una pérdida económica para las sociedades y países tener a jóvenes desempleados o inactivos.

De acuerdo a la información presentada, se puede categorizar una segunda forma de entender a la juventud en términos de un 'joven ocioso'. En este caso, el joven se transforma en un 'otro' desde la inactividad económica, es decir es el sujeto que no produce. $\mathrm{El}$ argumento de la OIT para la clasificación de este 'otro' como ocioso se basa en el costo económico que significa la inactividad para los países y en cómo este joven inactivo que no produce se puede transformar en un excluido de manera muy similar al joven pobre. Sin embargo, la diferencia es que este joven ocioso es psicologizado de manera individualizada, asociado al consumo de drogas y al crimen.

\section{La juventud como 'generación perdida'}

En el informe TEM (2010), se presentan variaciones respecto a informes anteriores en las formas de conceptualizar a la juventud, se describe por primera vez a la juventud como 'generación perdida'. A continuación, se presenta una cita donde se explicita qué se está entendiendo por esta categorización:

En las economías desarrolladas y en algunas economías en desarrollo, las repercusiones de la crisis en los jóvenes se manifiestan, sobre todo, en el desempleo y los peligros sociales derivados de la falta de trabajo y la inactividad prolongada. Diversos estudios revelan que el ingreso al mercado de trabajo durante un período de recesión puede marcar para siempre a la generación de jóvenes afectados. Últimamente, además, preocupa que la crisis deje como legado una "generación perdida", integrada por jóvenes que se desvinculan por completo del mercado de trabajo. Encontrar y motivar a jóvenes que han perdido las esperanzas de construir un futuro productivo es una tarea costosa. Sin embargo, la alternativa de no hacer nada es más costosa aún, puesto que a ello debe sumarse el costo social, económico e incluso político. (OIT, 2010. p. 1)

De acuerdo a esta tercera cita, se explicita claramente cómo el desempleo ha afectado a las economías avanzadas, producto de la crisis. Este es un hecho que da cuenta de cómo, producto de la crisis económica, la problemática del empleodesempleo deja de ser un tema de los países pobres y en desarrollo. A partir de este informe, podemos ver cómo se empieza a universalizar el tema del desempleo, es decir, empieza a tener cada vez más como protagonistas a los jóvenes de las economías avanzadas.

La OIT entiende como 'generación perdida' a los jóvenes que se desvinculan por completo del mercado del trabajo. Al igual que en los informes TEM anteriores, se sigue planteando el problema del empleo-desempleo en términos de costo beneficio. Sin embargo, a partir de ahora, no solo desde lo costosos que son los efectos de la crisis en términos económicos y sociales, sino también desde costos en términos políticos. La OIT advierte que este costo puede ser mayor aún, si no se hace nada para revertir a esta situación.

Esta categoría permite ilustrar cómo se construye una tercera forma de entender la juventud: 'la generación perdida'. La OIT habla 
de una población juvenil que es necesario 'guiar', ya que se encuentra 'perdida'. Vemos que aparece nuevamente la lógica del pastorado (Foucault, 2009), si bien es de forma distinta a la figura del joven pobre, se puede sostener que se mantiene una retórica orientada a conducir conductas de la juventud desde la OIT. La universalización del problema ha hecho que ya no se hable de jóvenes pobres. De hecho, en el informe TEM (2010), se plantea que la crisis no ha afectado a los países pobres como a los países industrializados, por lo que el discurso de erradicación de pobreza y los Objetivos del Mileno pierden protagonismo y deja de ser una bandera de lucha para la OIT. A partir de ahora, se habla de una 'generación perdida' que al igual que los 'jóvenes pobres', tiene que ser conducida para ser incluida en el mercado del trabajo. Frente a esto, es interesante detenerse en los sentimientos de urgencia que plantea la OIT para incluir a la generación perdida en el mercado del trabajo, sin embargo, en un contexto de recesión económica, no existe claridad por parte de los organismos internacionales de cómo hacer esto.

\section{La juventud feliz}

En el informe TEM (2010), se hace referencia a una juventud feliz e infeliz. Si bien estas conceptualizaciones tienen sus orígenes en documentos de las Naciones Unidas, la OIT las incorpora en el contexto donde se hacen visibles los efectos de la crisis económica:

Una juventud feliz es aquélla que se encuentra rodeada de apasionantes opciones para el futuro. Entonces, el contraste se presenta de la mano de una persona joven que se siente atrapada en una situación que ofrece pocas oportunidades de construir un futuro mejor. Aún más infelices son los jóvenes que tienen pocas esperanzas en cuanto a las perspectivas que depara el futuro y piensan que la desigualdad de su situación es injusta. Éstos son los jóvenes que se sienten víctimas del "sistema" y que vuelcan su enfado hacia el culpable que les resulta más evidente: la globalización en general, la avaricia del sistema capitalista, los políticos a nivel nacional, la corrupción del gobierno, sus padres o un grupo étnico específico, entre otros. (OIT, 2010. p. 58)

En esta cuarta cita, la conceptualización de 'juventud feliz' da cuenta de una forma de entender a los jóvenes, asociada al futuro, es decir, a un futuro mejor que un presente inmediato. El joven feliz obedece a un sujeto con múltiples opciones, que está proyectando su trayectoria laboral para ser funcional al mercado. Por otro lado, lo que podemos entender como 'la juventud infeliz' corresponde a una juventud que se siente 'victima' del sistema, no tiene proyecciones de futuro, y es infeliz en el presente, ya que piensa que la 'desigualdad de su situación es injusta'. Llama la atención la forma en que se construye a estos jóvenes como responsables de su infelicidad desde una perspectiva política, atribuyendo su enfado a la percepción que tienen de un modelo económico y político asociado a la globalización y al capitalismo. Hablar de felicidad e infelicidad en estos términos hace referencia a aspectos morales de la juventud, es decir, se construye una normatividad desde un 'deber ser' juvenil, donde el 'otro' que aparece como el 'joven infeliz' es asociado a un sujeto político que piensa en la 'desigualdad' y en la 'injusticia'. El joven infeliz es entendido entonces, como una figura politizada que busca culpables de su situación desde un cuestionamiento al sistema económico y político.

\section{Los desafortunados jóvenes}

Esta quinta categoría se desarrolla en el informe TEM (2013). En términos genéricos, este informe se plantea desde una posición más derrotista respecto a la juventud que los informes anteriores. Se pueden constatar una serie de conceptualizaciones de negatividad como 'jóvenes desafortunados', 'jóvenes desconfiados'. Esta forma de entender a la juventud, se basa principalmente en términos de la 'dificultad de ser joven' en el contexto actual. A esto se incorpora la variable azar, como una nueva forma de entender la problemática del empleodesempleo. En la siguiente cita que se presenta 
a continuación, se pueden ejemplificar estas afirmaciones derrotistas de la OIT:

No es fácil ser joven en el mercado de trabajo actual

El debilitamiento de la recuperación mundial en 2012 y 2013 ha agravado la crisis del empleo juvenil, dificultando aún más el acceso al empleo para muchos desafortunados jóvenes que buscan trabajo. Y está dificultándolo hasta tal punto que muchos están renunciado a seguir buscando. La prolongada crisis económica también obliga a la generación actual de jóvenes a ser menos selectivos con los empleos que están dispuestos a aceptar, una tendencia que ya era evidente antes de la crisis. El número de jóvenes que está aceptando trabajos a tiempo parcial o que se encuentra confinado en empleos temporales es cada vez mayor. Los empleos seguros, que en una época eran lo habitual para generaciones anteriores - por lo menos en las economías avanzadas - han pasado a ser más difíciles de conseguir para los jóvenes de hoy. (OIT, 2013. p. 1)

Esta quinta cita plantea la dificultad de ser joven y buscar trabajo en un contexto de post crisis económica. Esto, principalmente producto de la débil recuperación de los mercados, donde cada vez es más difícil acceder a empleo para la juventud. Se habla de 'desafortunados jóvenes' que 'buscan trabajo', y se explicita una preocupación desde la OIT por aquellos que definitivamente han dejado de buscar empleo. Por otro lado, el hecho de que los jóvenes sean menos selectivos con sus empleos favorece 'trabajos a tiempo parcial', es decir, valida prácticas de precariedad laboral contra los cuales había luchado la OIT por décadas. En esta línea, los 'empleos seguros', característicos de las economías avanzadas y propios del Estado de bienestar, son cada vez menos y por lo tanto difíciles de conseguir. La categorización 'los jóvenes desafortunados' da cuenta de una juventud con mala suerte por el momento histórico que le tocó vivir. El hecho de incorporar la variable azar para explicar el fenómeno de empleo-desempleo, podemos entenderlo como una pérdida de control de la situación por parte de organismos internacionales. Por otro lado, la escases del empleo y la ausencia de 'trabajo seguro' en tiempo de recuperación económica, ha validado una lógica economista de oferta y demanda, donde la precariedad y la flexibilización laboral se hacen cada vez más presentes en el mercado del trabajo. Siendo la más afectada por todo lo anterior, una población importante de 'desafortunados jóvenes' que buscan trabajo.

\section{Los jóvenes desconfiados}

En el mismo informe 2013, la OIT plantea una serie de costos y 'cicatrices' que ha dejado la crisis económica en nuestra sociedad actual, y cómo los jóvenes, producto de los efectos en el desempleo, han comenzado a expresar su desconfianza en los sistemas socioeconómicos y políticos.

Como ya se analizó en la edición de 2010 del informe de las Tendencias Mundiales del Empleo Juvenil, hay que pagar un precio si se quiere acceder al mercado de trabajo en tiempos de crisis económica. Hemos aprendido mucho sobre las «cicatrices» producidas en el poder adquisitivo futuro y en las vías de transición en el mercado de trabajo (OIT, 2010). Aunque, tal vez, las cicatrices más importantes sean las de la desconfianza que la generación actual de jóvenes tiene en los sistemas socioeconómicos y políticos. Parte de esta desconfianza se ha manifestado en protestas políticas como los movimientos contra la austeridad en España y Grecia. (OIT, 2013. p. 2)

En esta sexta cita, se sigue hablando en términos economicistas de costo y beneficio en la forma de visualizar el problema del empleodesempleo. Ahora, orientados a que la juventud tiene que pagar un 'precio' por acceder al mercado del trabajo en tiempos de crisis. La OIT se plantea como una institución que ha aprendido sobre 'las cicatrices' que ha significado la crisis económica. Siendo la más grave la desconfianza de la población juvenil, que ya no cree en el sistema económico y político, hecho que ha derivado en una serie de expresiones de resistencia desde la juventud, manifestadas desde la articulación de movimientos sociales 
en España y Grecia. Como ya se ha señalado, desde la OIT y otros Organismos Internacionales, existe la preocupación de que estos 'desconfiados jóvenes' dejen de creer en el sistema económico actual, transformándose en una amenaza clara al sistema económico y político.

Las seis figuras de la subjetividad del joven trabajador

Las categorías presentadas, podemos entenderlas como seis figuras de la subjetividad desde la cuales se construye al joven trabajador por la OIT. Todas estas figuras se estructuran de una forma dicotómica: a) joven pobre-no pobre, b) joven ocioso-joven productivo, c) generación perdida-generación no perdida, d) juventud felizinfeliz, e) jóvenes desafortunados-afortunados, y e) jóvenes desconfiados-confiados. Estas formas binarias incluyen una serie de elementos normativos que permiten la construcción de un adulto productivo en las sociedades actuales. Como se ha señalado, esta forma de clasificar a la juventud involucra una serie de elementos morales, basados en una lógica economicista, que se construye desde valores y principios neoliberales (Dean, 1995; Holmqvist et al., 2012; Fejes, 2010; Foucault, 2012).

De acuerdo a esta normatividad orientada a la producción, se puede dar cuenta de que los elementos planteados en las seis figuras de la subjetividad, no se basan en un 'deber ser' caracterizado por un 'ideal de joven trabajador', sino más bien, se construyen desde el hecho de que el joven debe evitar ser categorizado como un 'otro', un desconfiado, un desafortunado, un ocioso. Para la OIT el joven trabajador debe evitar transformarse en el excluido, su inclusión social y económica depende de no cruzar el umbral que lo transforma en un 'otro'. Por lo tanto, el joven trabajador debe ser un joven funcional que se transforma en un adulto productivo para un sistema económico que permite un orden global.

Desde los resultados obtenidos, vemos tres claras similitudes en estas seis figuras de la subjetividad. En primer lugar, se ha dado cuenta de que el joven debe ser guiado desde una lógica de pastorado (Foucault, 2009), ya sea para salir de su condición de 'joven pobre' o en su condición de 'generación pérdida'. Esta forma de entender cómo se gobierna la población juvenil, desde la conducción de la conducta orientada hacia el trabajo, permite entender la construcción del adulto productivo que espera formar el modelo neoliberal. En segundo lugar, los elementos positivos y características favorables asociadas a la juventud se construyen en el futuro más que en el presente. Tal como se ha mencionado en los primeros informes TEM (2004, 2006), se plantea una posición esperanzadora respecto a la juventud, definiéndola como el futuro de nuestras sociedades. Esta misma idea se ha podido ver en la categorización de la 'juventud feliz', ya que ambas conceptualizaciones no son planteadas en un presente inmediato, sino en proyecciones de futuro, basadas en cómo el joven contribuirá productivamente a la sociedad en su rol de adulto. En tercer lugar, podemos dar cuenta que a partir del año 2013, la OIT se ha posicionado desde una visión derrotista para plantear el problemática del empleo-desempleo. Este hecho ha connotado negativamente a la juventud, describiendo al joven trabajador como 'desafortunado' y 'desconfiado'. Estas formas de conceptualizar a la juventud están asociadas a respuestas que podríamos entender como inesperadas para los organismos internaciones, producto de una serie de manifestaciones políticas que emergen desde la ciudadanía organizada, en respuesta a la crisis y a las políticas de neoliberalización que desarrollan a nivel global.

A partir del análisis presentado, podemos constatar cómo el discurso inicial de la OIT, previo a la crisis, se sostiene desde un compromiso institucional. Este discurso se basa en una retórica que toma como referente la historia de la OIT como organización garante de derechos desde 1919, la cual, antes de la crisis, está claramente comprometida con la superación de la pobreza desde "un nuevo sentimiento de urgencia a nivel mundial para buscar soluciones a las dificultades de empleo de los jóvenes" (primera cita). Sin embargo, posterior a la crisis económica, esta forma de 
describir a la juventud cambia radicalmente. Como se ha mencionado, el joven empieza a ser connotado negativamente en los discursos. Además, el posicionamiento institucional de la OIT deja de basarse en el compromiso. Es más, a partir del informe TEM (2010), se desprende su compromiso histórico, planteando que frente a las variaciones del mercado, no es mucho lo que puede aportar en este contexto de recesión económica. A partir de esto, conceptualiza a la juventud que busca empleo en estos días como 'desafortunada', puesto que "no es fácil ser joven en el mercado del trabajo actual" (quinta cita). Este cambio discursivo se puede asociar a la 'teoría de las atribuciones' 7 que nos permitiría ejemplificar las posiciones tomadas por la OIT, entendiendo que el actor, en este caso la OIT, inicialmente asume un 'locus de control interno' en relación a la problemática, basada en el compromiso y la urgencia de un organismo internacional activo que contribuye a la generación de empleo desde la promoción del trabajo decente. Sin embargo, si hacemos una comparación con la posición asimilada posterior a la crisis económica, la OIT se desprende del problema, ya que lo sitúa como algo externo y propio del mercado. En este caso, podríamos entender la posición de la OIT desde una atribución de locus externo, donde los 'desafortunados jóvenes' tienen que adaptarse a un mercado precario y flexible que no garantiza la oportunidad del trabajo, ni las condiciones mínimas basadas en el 'trabajo decente'.

\section{Reflexiones Finales}

El presente estudio se ha preguntado cómo se construye al joven trabajador desde una perspectiva global, para lo cual se han tomado como referencia las conceptualizaciones de la OIT, debido a su importancia como entidad reguladora del trabajo en el mundo. A partir del análisis realizado de los informes TEM, se han podido constatar una serie de formas en las cuales se clasifica normativamente al joven trabajador. Se ha ilustrado cómo emergen, al menos seis figuras de la subjetividad en la última década.
A su vez, se han dado luces de las variedades discursivas y las distintas posiciones que la OIT ha asumido en relación al empleo-desempleo juvenil. A continuación, se desarrollan dos ideas finales para cerrar este estudio.

En primer lugar, se ha podido constatar cómo en los últimos años, se ha instalado una racionalidad Neoliberal en los discursos de la OIT en relación al trabajo. Vemos cómo está presente de manera transversal en todos los informes, la idea economicista de costo-beneficio para entender la problemática del empleodesempleo juvenil. Desde esta perspectiva, el hecho de que el joven se transforme en un sujeto trabajador o en un adulto productivo, es parte de un 'contrato social' que algunos autores han denominado como el 'orden social del trabajo' (Hopenhaym, 2001; Ibáñez, 1996; Medá, 1998). De acuerdo con lo revisado en este artículo, podemos sostener entonces, que corresponde a una forma de gobierno de la población juvenil, que permite un orden global. Esto significa que el joven debe transformarse en un 'adulto productivo' como parte de su rol social, por lo tanto, debe reconocer e incorporar este mandato social en su comportamiento, y hacerlo parte de su subjetividad. Si el joven no cumple o rompe con este mandato social, se convierte en 'otro', en un excluido, en el diferente que puede adquirir una forma individual $\mathrm{y} / \mathrm{o}$ colectiva. En segundo lugar, desde la perspectiva de la Gubernamentalidad, se puede sostener que las técnicas de gobierno orientadas a la juventud y el trabajo están asociadas a la inestabilidad mercado. De acuerdo a lo revisado en este artículo, se puede argumentar que la OIT está produciendo categorías y formas de entender al joven trabajador cada dos años. Hemos revisado cómo en la última década, las formas en que emergen las figuras de la subjetividad se establecen, cambian y posteriormente son olvidadas. Un ejemplo de esto es la figura del 'joven pobre', que en los primeros informes tiene mucha fuerza y sostiene un discurso institucional, sin embargo, en los últimos años este joven pobre desaparece junto a los Objetivos del Milenio y la priorización del tercer mundo en relación al desempleo juvenil. Actualmente, las figuras de la 
subjetividad están centradas más bien en 'el joven desafortunado' y 'el joven desconfiado', producto de las variaciones del mercado y la respuesta política de la juventud frente a los efectos de la crisis económica.

Finalmente, hemos podido constatar cómo estas figuras de la subjetividad, elaboradas desde la OIT, permiten prescribir formas de conducir la conducta de los jóvenes para su inclusión en el mercado del trabajo. En esta línea, para que estas tecnologías de gobierno (Foucault, 2012b; Castro-Gómez, 2010) sigan siendo eficientes, las conceptualizaciones del joven trabajador seguirán mutando al ritmo de un mercado cambiante. Los resultados del presente estudio dejan abierta la posibilidad de encontrar nuevas figuras en de la subjetividad en futuros informes TEM. Por ahora, dentro del marco de la Gubernamentalidad, se dejan los siguientes interrogantes iserá posible entender el problema de la construcción del joven trabajador desde otra perspectiva que no se base exclusivamente en una racionalidad económica? (Foucault, 2012; Holmqvist et al., 2012; Garsten \& Jacobsson, 2013; Rivera-Aguilera, 2016) iSerá posible gobernar a la juventud desde otras formas de entender las políticas del trabajo?

\section{Referencias}

Ainsworth, S. (2001). The discursive construction of older worker identity: A reflection on process and methods. Tamara Journal of critical postmodern Organization Science, 1(4), 29-47.

Assusa, G., \& Brandán, M. G. (2014). "Salvar la generación perdida": Gubernamentalidad, empleabilidad y cultura del trabajo. El caso de un programa de empleo para jóvenes en Argentina. Revista Sociología e Política, 22, 157-174. https://doi.org/10.1590/S0104-44 782014000100009

Atkinson, P., \& Coffey, A. (2006). Analysing documentary realities. En D. Silverman (Ed.), Interpreting Qualitative Data (pp. 56-75). Londres: Sage.
Brandán, M. G. (2014). Juventud, trabajo y dispositivos estatales. Aportes críticos a la sociología de la juventud desde la perspectiva de la Gubernamentalidad. Última Década, 40(1), 37-54. https://doi.or g/10.4067/S0718-22362014000100003

Burchell, G., Gordon, C., \& Miller, P. (1991). The Foucault effect: Studies in Governmentality. Chicago, IL: The University of Chicago press.

Castro-Gómez, S. (2010). Historia de la Gubernamentalidad. Razón de Estado, liberalismo y neoliberalismo en Michel Foucault. Bogotá: Siglo del Hombre Editores.

Chertkovskaya, E., Watt, P., Tramer, S., \& Spoelstra, S. (2013). Giving notice to employability. Ephemera, 13(4), 701-716.

Dean, M. (1995). Governing the unemployed self in an active society. Economy and Society, 24(4), 559-583. https://doi.org/10.1080/03 085149500000025

Diedrich, A., \& Styhre, A. (2013). Constructing the employable immigrant: The uses of validation practices in Sweden. Ephemera, 13(4), 759-783.

Du Gay, P. (1996). Consumption and identity at work. Londres: Sage.

Fairclough, N., \& Wodak, R. (2000). Análisis crítico del discurso. En T. Van Dijk (Ed.), El discurso como interacción social (pp. 367-404). Barcelona: Gedisa.

Fairclough, N. (2010). Critical Discourse Analysis. The critical study of language. Londres: Longman.

Fejes, A. (2010). Discourses on employability: Constituting the responsible citizen. Studies in continuing education, 32 (2), 89-102. https ://doi.org/10.1080/0158037X.2010.488353

Flew, T. (2014). Six theories of neoliberalism. Thesis Eleven, 122(1), 49-71. https://doi.org /10.1177/0725513614535965

Foucault, M. (2009). Seguridad, territorio y población. Buenos Aires: Fondo de Cultura Económica.

Foucault, M. (2012). El Nacimiento de la Biopolítica. Buenos Aires: Fondo de Cultura Económica. 
Foucault, M. (2012b). Tecnologías del Yo y otros textos a fines. Barcelona: Paidós.

Garsten, C., \& Jacobsson, K. (2013). Sorting people in and out: The plasticity of the categories of employability, work capacity and disability as technologies of government. Ephemera, 13(4), 825-850.

Grinberg, S. (2007). Gubernamentalidad: estudios y perspectivas. Revista Argentina de Sociología, 5(8), 95-110. Recuperado desde http://www.redalyc.org/articulo.oa?i $d=26950806$

Holmqvist, M., Maravelias, C., \& Skalen, P. (2012). Identity regulation in neo-liberal societies: Constructing the "occupationally disabled" individual. Organization, 20(2), 193-211. https://doi.org/10.1177/13505084 12438704

Hopenhayn, M. (2001). Repensar el trabajo. Buenos Aires: Norma.

Hopman, M., Winter, M., \& Koops, W. (2014). The hidden curriculum of youth policy: a Dutch example. Youth Eु Society, 46(3), 360-378. https://doi.org/10.1177/0044118x 11436187

Ibáñez, T. (1996). Fluctuaciones Conceptuales: en torno a la Postmodernidad y la Psicología. Caracas: Universidad Central de Venezuela.

Ibáñez, T. (2004). Introducción a la psicología social. Barcelona: UOC.

Kaasch, A. (2013). Contesting contestation: Global social policy prescriptions on pensions and health systems. Global Social Policy, 13(1), 45-65. https://doi.org/10.1177 /1468018112469797

Medá, D. (1998). El trabajo. Un valor en peligro de extinción. Barcelona: Gedisa.

Miller, P., \& Rose, N. (2009). Governing the present. Cambridge: Polity Press.

Mussetta, P. (2009). Foucault y los anglofocultianos: una reseña del Estado y la Gubernamentalidad. Revista Mexicana de Ciencias Políticas y Sociales, LI(205), 37-55.

Organización Internacional del Trabajo, OIT. (2004). Tendencias mundiales del empleo juvenil. ISBN: 92-2-315998-9. Recuperado de http://www.ilo.org/empelm/pubs/WCM _041933/lang--es/index.htm

Organización Internacional del Trabajo, OIT. (2006). Tendencias Mundiales del empleo juvenil. ISBN: 92-2-318627-7. Recuperado de http://www.ilo.org/empelm/pubs/WCM _041931/lang--es/index.htm

Organización Internacional del Trabajo, OIT. (2008). Tendencias Mundiales del empleo juvenil. ISBN: 978-92-2-321544-6. Recuperado de http://www.ilo.org/empelm/ pubs/WCMS_114358/lang--es/index.htm

Organización Internacional del Trabajo, OIT. (2010). Tendencias Mundiales del empleo juvenil. ISBN: 978-92-2-323856-8. Recuperado de http://www.ilo.org/empelm/ pubs/WCMS_150034/lang--es/index.htm

Organización Internacional del Trabajo, OIT. (2012). Tendencias Mundiales del empleo juvenil ISBN: 978-92-2-126325-8. Recuperado de http://www.ilo.org/global/re search/global-reports/youth/2012/WCMS 180976/lang--es/index.htm

Organización Internacional del Trabajo, OIT. (2013). Tendencias Mundiales del empleo juvenil. ISBN: 978-92-2-327484-9 Recuperado de http://www.ilo.org/wcmsp5/ groups/public/-.-dgreports/-.-dcomm/docu ments/publication/wcms_222658.pdf

Organización Internacional del Trabajo, OIT. (2014). Tendencias Mundiales del empleo juvenil. Recuperado de http://www.ilo.org/global/research/globa 1-reports/global-employment-trends/2014/ WCMS 234000/lang--es/index.htm

Organización Internacional del Trabajo, OIT. (2017). Misión e impacto de la OIT. Ginebra, Suiza: Organización Internacional del Trabajo. Recuperado de http:/www.ilo.org/global/about-the-ilo/ mission-and-objectives/lang--es/index.htm

Parker, I. (2012). Discourse Analysis: Dimensions of Critique in Psychology. Qualitative Research in Psychology, 10(3), 223-239. http s://doi.org/10.1080/14780887.2012.741509

Parker, I. (2013). Micronaciones del sí-mismo en tiempos de guerra: análisis de discurso 
y psicología. Universitas Psychologica, 12(1), 301-312.

Prior, L. (2006). Doing things with documents. En D. Silverman (Ed.), Interpreting Qualitative Data. Londres: Sage.

Prior, L. (2008). Repositioning documents in social research. Sociology, 42 (5), 821-836. h ttps://doi.org/10.1177/0038038508094564

Pulido-Martínez, H. C. (2008). Produciendo trabajadores modernos. Conocimiento psicológico y el mundo del trabajo en el sur. Universitas Psychologica, 6(1), 27-37.

Pulido-Martínez, H. C. (2012). El trabajador libre y la psicología del trabajo. En S. Trujillo-García, \& H. C. Pulido-Martínez (Eds.), Libertad y psicología. Tensiones y debates desde Iberoamérica (pp.171-188). Bogotá: Editorial Pontificia Universidad Javeriana.

Pulido-Martínez, H. C., \& Sato, L. (2013). ... Y entonces iesto de la crítica qué es? De las relaciones entre la psicología y el mundo del trabajo. Universitas Psychologica, 12(4), 1355-1368. https://doi.org/10.11144/Javeri ana.upsy12-4.rpmt

Rivera-Aguilera, G. (2016). Gubernamentalidad y Políticas de empleo: La construcción discursiva del joven trabajador en Chile. Última Década, 45(2), 34-54. https://doi.or $\mathrm{g} / 10.4067 / \mathrm{S} 0718-22362016000200003$

Rivera-Aguilera, G. (2017). Los procesos de influencia global/local en políticas públicas: Una propuesta metodológica. Psicoperspectivas, 16(3), 111-121. https://doi.org/10.5027/psicopers pectivas-vol16-issue3-fulltext-1092

Rose, N. (1996). Inventing our selves. Psychology, power and personhood. Cambridge: Cambridge University Press.

Rose, N., O’ Malley, P., \& Valverde, M. (2012). Gubernamentalidad. Astrolabio, 8, 113-152.

Sáenz, J., \& Granada, C. (2013). El dispositivo de lo social como gobierno de los pobres en la primera mitad del siglo XX en Colombia. En O. Restrepo (Ed.), Proyecto ensamblando Colombia (Tomo 1, pp. 219-252). Bogotá: Universidad Nacional de Colombia.
Senellart, M. (2009). Situación de los cursos. En M. Foucault (Ed.), Seguridad, territorio y población (pp. 417-453). Buenos Aires: Fondo de Cultura Económica.

Sennett, R. (2013). La corrosión del carácter. Barcelona: Anagrama.

Sisto, V. (2012). Identidades desafiadas: individualización, managerialismo y trabajo docente en el Chile actual. Psykhe, 21(2), 35-46. https://doi.org/10.7764/psykhe.21.2. 542

Sisto, V., \& Fardella, C. (2011). Nuevas políticas públicas, epocalismo e identidad: el caso de las políticas orientadas a los docentes en Chile. Revista de Estudios Universitarios, 37(1), 123-141.

Stecher, A. (2010). El análisis crítico del discurso como herramienta de investigación psicosocial del mundo del trabajo. Discusiones desde América Latina. Universitas Psychologica, 9(1), 93-107. Recuperado de http://revistas.javeriana.edu.co/index.p $\mathrm{hp} /$ revPsycho/article/view/308/426

Stecher, A. (2014). Fairclough y el lenguaje en el Nuevo Capitalismo: Análisis de las dimensiones discursivas del mundo del trabajo. Psicoperspectivas, 13(3), 19-29.

Stecher, A., \& Godoy, L. (2014). Transformaciones del trabajo, subjetividad e identidades. Lecturas psicosociales desde Chile y América Latina. Santiago de Chile: Ril Editores.

Vargas-Monroy, L., \& Pujal, M. (2013). Gubernamentalidad, dispositivos de género, raza y trabajo: la conducción de la conducta de las mujeres trabajadoras. Universitas Psychologica, 12(4), 1255-1267. https://doi.org/10.11144 /Javeriana.upsy12-4.gdgt

Vesterberg, V. (2013). Ethnicized un/ employability: Problematized others and the shaping of advanced liberal subjetcs. Ephemera, 13(2), 737-757. 


\section{Notas}

1 Los datos oficiales de la OIT (2013) señalan que hay 73 millones de jóvenes entre 15 y 24 años desempleados.

2 El Grupo de los 20 es un foro de 19 países, más la Unión Europea. Desde 1999 estudia, revisa y promueve discusiones sobre temas relacionados con los países industrializados y las economías emergentes con el objetivo de mantener la estabilidad financiera internacional.

3 La Organización Internacional del Trabajo (OIT) está consagrada a promover la justicia social y los derechos humanos y laborales reconocidos a nivel internacional. Su misión fundadora: la paz laboral es esencial para la prosperidad.

4 Se define como una fuente de asistencia financiera y técnica para los llamados países en desarrollo. Su propósito declarado es reducir la pobreza mediante préstamos de bajo interés, créditos sin intereses a nivel bancario y apoyos económicos a las naciones en desarrollo. Está integrado por 188 países miembros y depende de las Naciones Unidas.

5 Movimiento 15-M, también llamado movimiento de los indignados, fue un grupo ciudadano formado por jóvenes que produjo una serie de protestas pacíficas en España durante el año 2011.

6 Actualmente ha habido modificaciones en los objetivos de la OIT. Los que se plantean acá corresponden a la información recogida durante los años 2014-2015.

7 La Teoría de la atribución: es una teoría de la psicología social desarrollada inicialmente por el psicólogo Fritz Heider (1958) The Psychology of Interpersonal Relations (Ibáñez, 2004).

* Artículo de investigación, elaborado durante pasantía en la Facultad de Ciencias Sociales de la Pontificia Universidad Javeriana de Bogotá, Colombia. Agracedimiento a la beca 'Alianza Pacífico' cuyo financiamiento permitió la estancia en este centro de estudios durante el primer semestre del 2015. 